\title{
EL SISTEMA ALIMENTARIO COMO DINAMIZADOR DE ÁREAS DEMOGRÁFICAMENTE DEPRIMIDAS: EL CASO DE LA INDUSTRIA LÁCTEA
}

\author{
Alicia Langreo Navarro ${ }^{\mathrm{a}}$ y Tomás García Azcárate ${ }^{\mathrm{b} *}$, \\ ${ }^{a}$ Directora de Sabora, S.L. E-mail: alangreonavarro@gmail.com ${ }^{b}$ Vice-Director del Instituto de \\ Economía, Geografía y Demografía (IEGD-CSIC) e investigador asociado del CEIGRAM, Madrid, E-mail: \\ tomasgarciaazcarate@gmail.com
}

Resumen: Nos referimos como sistema alimentario el conjunto de empresas cuyo concurso es imprescindible para la producción de alimentos. Abarca la producción primaria, la comercialización mayorista en origen y destino, la industria de primera y segunda transformación e incluso podría incluirse la distribución minorista; además del suministro (y en su caso fabricación) de insumos, los servicios logísticos, la asesoría en sus múltiples versiones o los servicios públicos vinculados. Anteriormente, hemos defendido que el sistema alimentario es el principal motor socioeconómico en la mayor parte de las áreas rurales. Aquí hacemos una aproximación de su aportación (en términos de PIB, ocupación, inversión y compras de materias primas) a los territorios españoles con problemas poblacionales (caída de la población, baja densidad, desequilibrios demográficos), para lo que utilizamos diversas fuentes estadísticas: Encuesta Industrial, Estadísticas agrarias, Padrón y Censo de la Población. El ámbito geográfico y la cadencia condicionan el manejo de estas fuentes; se utiliza tanto la comunidad autónoma, como la provincia y, para la variable de ocupación, el rango del municipio según dimensión poblacional. Las limitaciones estadísticas no permiten contemplar los servicios al sistema alimentario ni los insumos, pero si la aportación de las fases primaria, la comercial mayorista y la industrial.

Palabras claves: Industria alimentaria, España rural, España vaciada

\section{1.- Introducción y metodología}

Esta comunicación entiende como sistema alimentario el conjunto de empresas cuyo concurso es imprescindible para la producción de alimentos. Abarca la producción primaria, la comercialización mayorista en origen y destino, la industria de primera y segunda transformación e incluso podría incluirse la distribución minorista; además del suministro (y en su caso fabricación) de insumos, los servicios logísticos, la asesoría en sus múltiples versiones o los servicios públicos vinculados. En anteriores trabajos hemos defendido que el sistema alimentario es el principal motor socioeconómico en la mayor parte de las áreas rurales y en general en las zonas con baja densidad de población, pero la traducción a cifras de este planteamiento es difícil debido a que las fuentes estadísticas no están preparadas ni para el tratamiento global del sistema alimentario ni para descender al medio rural. Por eso hemos hecho diversas aproximaciones parciales, en esta comunicación nos centramos en la industria de primera transformación, concretamente la industria láctea. Queremos aclarar que esta comunicación forma parte de una línea de trabajo encaminada a ir aclarando las diferentes facetas de esta cuestión.

El objetivo es hacer una aproximación a la aportación a la economía local en términos de PIB, ocupación, inversión y compras de materias primas) en los territorios españoles con problemas poblacionales (caída de la población, baja densidad, desequilibrios demográficos). Estas variables referidas a la industria alimentaria se encuentran en la Encuesta Industrial del INE, pero el ámbito geográfico es la comunidad autónoma, por lo tanto se analiza el comportamiento de las mismas en aquellas comunidades que presentan indicadores demográficos peores que la media española. Este ámbito indudablemente presenta distorsiones y no permite centrarnos en exclusiva en el medio rural, pero hay que tener en cuenta que, especialmente en las comunidades menos pobladas y con menos dinamismo económico, la capacidad de arrastre de la industria supera ampliamente a la comarca y se deja sentir a todos los efectos en todo el ámbito territorial de referencia.

Se ha desechado el tratamiento conjunto de la industria alimentaria, ya que la concentración de la industria de segunda transformación y en parte de la industria vinculada a importaciones se concentra en grandes núcleos industriales y enmascara la importancia real de la industria alimentaria en dichos territorios.

En el desarrollo del trabajo nos hemos encontrado el problema de la falta de información subsectorial más allá del año 2015; por eso hemos utilizado esas cifras, últimas disponibles.

El trabajo se complementa con información del registro de establecimientos industriales de la Agencia de Seguridad Alimentaria sobre la presencia de industrias en municipios de pequeño tamaño. Este tratamiento tiene el inconveniente de que no hace ninguna referencia a la dimensión de las mismas. 


\section{2.- El planteamiento}

Gran parte del interior peninsular se enfrenta a graves problemas poblacionales, con escasa densidad, desequilibrios poblacionales, falta de capital humano para el desarrollo y falta de núcleos de población que pueda sostener una mínima dinámica económica y social. En nuestra opinión la causa de esta situación es el hundimiento de los sistemas tradicionales de producción, que no han sido sustituidos por otros. Este deterioro provoca que este medio resulte poco competitivo para atraer o retener inversiones y capital humano. A eso se suma la falta de determinadas infraestructuras.

Sin embargo, en este entorno hay sistemas económicos que se muestran dinámicos, están basados en los recursos agrícolas y ganaderos y, en general son verdaderos motores económicos en sus territorios. Entre ellos se encuentra la ganadería intensiva de carne y leche.

La existencia del sistema lácteo de producción aporta a los territorios actividad en ganadería y producción y venta de forrajes y piensos, servicios a la ganadería (veterinaria, gestoría, suministros y mantenimiento de maquinaria e instalaciones, etc.), comercio de animales vivos, logística, centros de recogida de leche, industria y servicios a la industria.

\section{3.- Importancia de la industria láctea en las comunidades con problemas poblacionales}

Las principales conclusiones en esta cuestión son:

- En torno a un $30 \%$ del personal, las ventas y las compras de materias primas y casi el $40 \%$ de la inversión se realizan en CCAA con menos de $30 \mathrm{hb} / \mathrm{km} 2$

- Casi el $60 \%$ del personal, de las compras de materias primas y de la inversión y casi el $70 \%$ de las ventas se realizan en CCAA que han perdido población desde 2009.

Cuadro 1. La importancia de la industria láctea en las CCAA con pérdida de habitantes

\begin{tabular}{|l|l|l|l|}
\hline Pérdida de habitantes & $>5 \%$ & $0-5 \%$ & Total \\
\hline$\%$ Establecimientos & $15,22 \%$ & $29,49 \%$ & $44,71 \%$ \\
\hline$\%$ Personal & $27,18 \%$ & $32,66 \%$ & $59,84 \%$ \\
\hline$\%$ Ventas & $29,78 \%$ & $37,53 \%$ & $67,31 \%$ \\
\hline $\begin{array}{l}\% \text { Compras materia } \\
\text { prima }\end{array}$ & $18,26 \%$ & $38,54 \%$ & $56,84 \%$ \\
\hline$\%$ Inversión & $29,52 \%$ & $0,44 \%$ & $58,15 \%$ \\
\hline
\end{tabular}

Fuente: Padrón municipal (2009-2019) y Encuesta de Estructuras 2015 (INE)

Además hay que resaltar:

- En las 37 provincias en las que ha caído la población y/o la densidad es inferior a $75 \mathrm{hb} / \mathrm{km} 2 \mathrm{se}$ encuentra casi la mitad de los establecimientos

- Más del 23\% de los establecimientos están en provincias que han perdido más del 5\% de su población entre 2009 y 2019

- Más del 30\% de los establecimientos está en provincias que tienen una densidad de menos de $50 \mathrm{hb} / \mathrm{km} 2$

Cuadro 2. La importancia de la industria láctea en las CCAA con menos densidad poblacional

\begin{tabular}{|l|l|l|l|}
\hline Densidad & $<30 \mathrm{hb} / \mathrm{km} 2$ & $30-75 \mathrm{Hb} / \mathrm{km} 2$ & $<75 \mathrm{hb} / \mathrm{km} 2$ \\
\hline \% Establecimientos & $27,48 \%$ & $3,70 \%$ & $31,18 \%$ \\
\hline$\%$ Personal & $31,96 \%$ & $1,76 \%$ & $33,72 \%$ \\
\hline$\%$ Ventas & $30,63 \%$ & $1,66 \%$ & $34,19 \%$ \\
\hline $\begin{array}{l}\% \text { Compras materia } \\
\text { prima }\end{array}$ & $32,50 \%$ & $1,69 \%$ & $34,19 \%$ \\
\hline \begin{tabular}{l} 
\% Inversión \\
\hline
\end{tabular} & $39,21 \%$ & $0,44 \%$ & $39,65 \%$ \\
\hline
\end{tabular}

Fuente: Padrón municipal (2009-2019) y Encuesta de Estructuras 2015 (INE)

4.- La localización de los establecimientos industriales lácteos en las provincias con mayores problemas poblacionales y en municipios de pequeño tamaño

Este análisis cuenta con el problema de que es imposible saber la dimensión de los establecimientos, es posible que muchas de las industrias que aparecen en municipios muy pequeños sean industrias artesanas queseras, aun así, su elevado número en los estratos de menor dimensión poblacional da una idea de la 
enorme capacidad de dinamización con que cuenta el sector. Cabe mencionar que también se han encontrado grandes fábricas en municipios de monos de 50.000 y 20.000 habitantes.

Las principales conclusiones al respecto son:

- Cerca del $40 \%$ de los establecimientos están en municipios de menos de 2.000 habitantes

- Cerca del 55\% en municipios de menos de 5.000 habitantes

- Casi el 70\% están en municipios de menos de 10 habitantes

- Menos del $10 \%$ están en ciudades de más de 50.000 habitantes

Asimismo, a partir del Registro de industrias de la AESAN se han localizado los establecimientos industriales por provincias, señalando su peso en aquellas con problemas poblacionales.

Cuadro 3. La concentración de los establecimientos de la industria láctea en las provincias con problemas poblacionales

\begin{tabular}{|l|l|l|l|l|}
\hline $\begin{array}{l}\text { Densidad de } \\
\text { población }\end{array}$ & $\mathrm{N}^{\mathrm{o}}$ Provincias & $\mathrm{N}^{\mathrm{o}}$ Establecimien & Establecimien & $\%$ Superficie \\
\hline$<20 \mathrm{hb} / \mathrm{Km} 2$ & 8 & 229 & 9,42 & 20,68 \\
\hline $20-50 \mathrm{hb} / \mathrm{Km} 2$ & 13 & 537 & 22,09 & 34,79 \\
\hline $50-75 \mathrm{Hb} / \mathrm{km} 2$ & 7 & 206 & 8,47 & 15,21 \\
\hline Total & 28 & 972 & 39,98 & 70,69 \\
\hline
\end{tabular}

Fuente: AESAN e INE

\section{5.- Conclusiones}

La industria láctea tiene una presencia considerable en los territorios con problemas poblacionales y las cifras de la distribución del empleo, las inversiones, el volumen de venta y la compra de materias primas en esas áreas ponen de manifiesto el rol que juegan. Las estadísticas disponibles no facilitan llegar mucho más allá e incluso de cara al futuro la falta de desagregación en subsectores de la Encuesta Industrial del INE en las comunidades autónomas dificultará más la continuación de los análisis.

\section{Bibliografía}

Bachmann, M.R. (1985): Milk Processing in Rural Areas to support Dairying in Developing Countries Journal of Dairy Science 68 (8): 2134-2139

Esparcia Pérez, J. (2003): Sectores productivos y dinámica socioeconómica. Una aproximación a la situación y cambios recientes en áreas rurales valencianas. Serie Geográfica 11: 91-115

Lambert, D.: McNamara, K.T.; Garett, M. I. (2006): Food Industry Investment Flows: Implications for Rural development The review of Regional Studies 36 (2): 140-162

Shearer, J.W. (1999): mapping the milk industry: Towards a comprehensive Geographical Information System International Jpurnal of dairy technology 32 (1): 1-11 
Mapa 1 y 2. Establecimientos de la industria láctea en provincias con problemas poblacionales
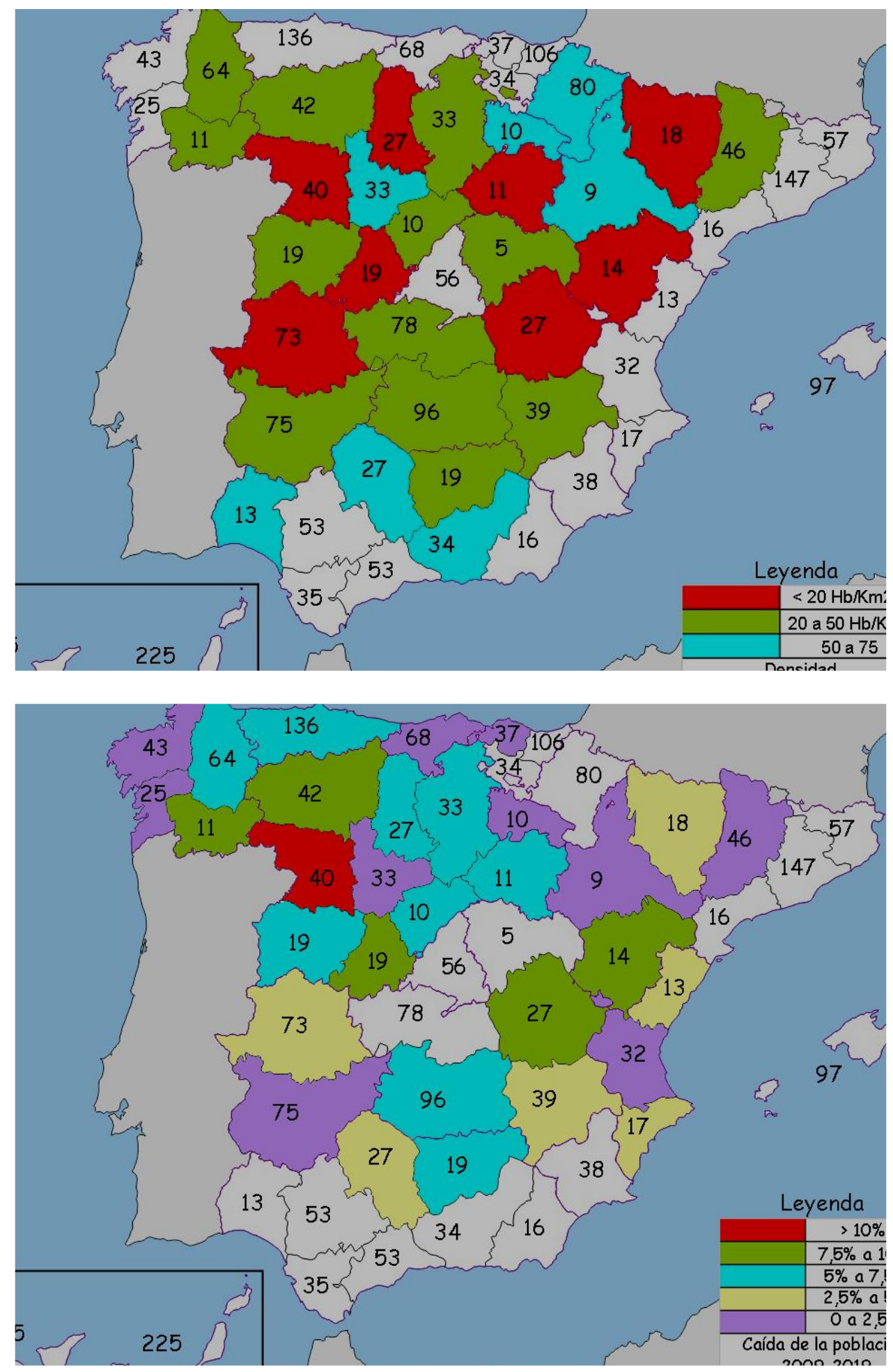